\title{
Water emission from the high-mass star-forming region IRAS 17233-3606 $\star$, $\star \star$
}

\section{High water abundances at high velocities}

\author{
S. Leurini ${ }^{1}$, A. Gusdorf ${ }^{2}$, F. Wyrowski ${ }^{1}$, C. Codella ${ }^{3}$, T. Csengeri ${ }^{1}$, F. van der Tak ${ }^{4,5}$, H. Beuther ${ }^{6}$, D. R. Flower ${ }^{7}$, \\ C. Comito ${ }^{8}$, and P. Schilke ${ }^{8}$ \\ ${ }^{1}$ Max-Planck-Institut für Radioastronomie, Auf dem Hügel 69, 53121 Bonn, Germany \\ e-mail: sleurini@mpifr.de \\ 2 LERMA, UMR 8112 du CNRS, Observatoire de Paris, École Normale Supérieure, 24 rue Lhomond, 75231 Paris Cedex 05, France \\ 3 INAF - Osservatorio Astrofisico di Arcetri, Largo E. Fermi 5, 50125 Firenze, Italy \\ 4 SRON Netherlands Institute for Space Research, PO Box 800, 9700 AV Groningen, The Netherlands \\ 5 Kapteyn Astronomical Institute, University of Groningen, PO Box 800, 9700 AV Groningen, The Netherlands \\ ${ }^{6}$ Max-Planck-Institute for Astronomy, Königstuhl 17, 69117 Heidelberg, Germany \\ 7 Physics Department, The University, Durham DH1 3LE, UK \\ 8 Physikalisches Institut, Universität zu Köln, Zülpicher Str. 77, 50937 Köln, Germany
}

Received 27 December 2013 / Accepted 18 March 2014

\section{ABSTRACT}

\begin{abstract}
We investigate the physical and chemical processes at work during the formation of a massive protostar based on the observation of water in an outflow from a very young object previously detected in $\mathrm{H}_{2}$ and $\mathrm{SiO}$ in the IRAS $17233-3606$ region. We estimated the abundance of water to understand its chemistry, and to constrain the mass of the emitting outflow. We present new observations of shocked water obtained with the HIFI receiver onboard Herschel. We detected water at high velocities in a range similar to SiO. We self-consistently fitted these observations along with previous $\mathrm{SiO}$ data through a state-of-the-art, one-dimensional, stationary $\mathrm{C}$-shock model. We found that a single model can explain the $\mathrm{SiO}$ and $\mathrm{H}_{2} \mathrm{O}$ emission in the red and blue wings of the spectra. Remarkably, one common area, similar to that found for $\mathrm{H}_{2}$ emission, fits both the $\mathrm{SiO}$ and $\mathrm{H}_{2} \mathrm{O}$ emission regions. This shock model subsequently allowed us to assess the shocked water column density, $N_{\mathrm{H}_{2} \mathrm{O}}=1.2 \times 10^{18} \mathrm{~cm}^{-2}$, mass, $M_{\mathrm{H}_{2} \mathrm{O}}=12.5 M_{\oplus}$, and its maximum fractional abundance with respect to the total density, $x_{\mathrm{H}_{2} \mathrm{O}}=1.4 \times 10^{-4}$. The corresponding water abundance in fractional column density units ranges between $2.5 \times 10^{-5}$ and $1.2 \times 10^{-5}$, in agreement with recent results obtained in outflows from low- and high-mass young stellar objects.
\end{abstract}

Key words. stars: formation - stars: protostars - ISM: jets and outflows - ISM: individual objects: IRAS 17233-3606 astrochemistry - shock waves

\section{Introduction}

The formation mechanism of high-mass stars $\left(M>8 M_{\odot}\right)$ has been an open question despite active research for several decades now, the main reason being that the strong radiation pressure exerted by the young massive star overcomes its gravitational attraction (Kahn 1974). Controversy remains about how highmass young stellar objects (YSOs) acquire their mass (e.g., Krumholz \& Bonnell 2009), either locally in a prestellar phase or during the star formation process itself, being funnelled to the centre of a stellar cluster by the cluster's gravitational potential. Bipolar outflows are a natural by-product of star formation and understanding them can give us important insights into the way massive stars form. In particular, studies of their properties in terms of morphology and energetics as function of the luminosity, mass, and evolutionary phase of the powering object may help us to understand whether the mechanism of formation of

\footnotetext{
* Herschel is an ESA space observatory with science instruments provided by European-led Principal Investigator consortia and with important participation from NASA.

$\star \star$ Appendices are available in electronic form at http://www . aanda.org
}

low- and high-mass YSOs is the same or not (see, e.g., Beuther et al. 2002).

Water is a valuable tool for outflows as it is predicted to be copiously produced under the type of shock conditions expected in outflows (Flower \& Pineau Des Forêts 2010). Observations of molecular outflows powered by YSOs of different masses reveal abundances of $\mathrm{H}_{2} \mathrm{O}$ associated with outflowing gas of the order of some $10^{-5}$ (e.g., Emprechtinger et al. 2010; Kristensen et al. 2012; Nisini et al. 2013). Recently, the Water In Starforming regions with Herschel (van Dishoeck et al. 2011) key program targeted several outflows from Class 0 and I low-mass YSOs in water lines. $\mathrm{H}_{2} \mathrm{O}$ emission in young Class 0 sources is dominated by outflow components; in Class I YSOs $\mathrm{H}_{2} \mathrm{O}$ emission is weaker because of less energetic outflows (Kristensen et al. 2012). Comparisons of low-excitation water data with $\mathrm{SiO}$, $\mathrm{CO}$, and $\mathrm{H}_{2}$ reveal contrasting results because these molecules seem to trace different environments in some sources (Nisini et al. 2013; Tafalla et al. 2013) while they have similar profiles and morphologies in others (Lefloch et al. 2012; Santangelo et al. 2012). Observations of massive YSOs (e.g., van der Tak et al. 2013) confirm broad profiles due to outflowing gas in low-energy $\mathrm{H}_{2} \mathrm{O}$ lines. However, the coarse spatial resolution of 


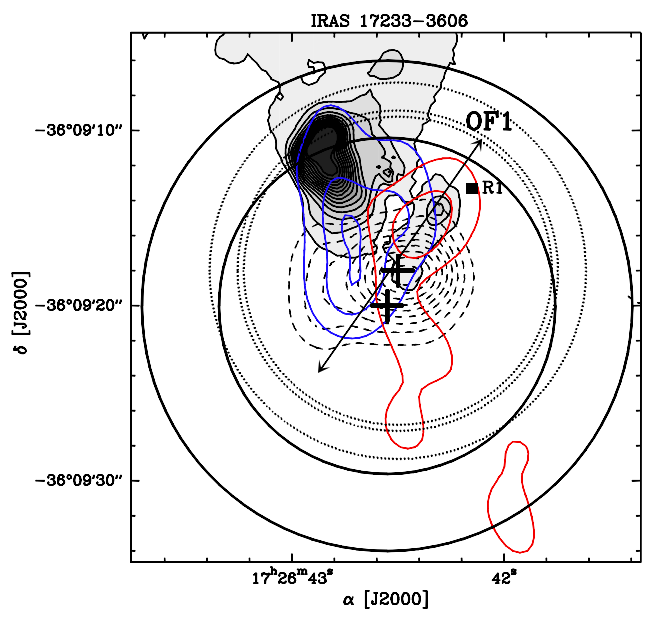

Fig. 1. Grey scale and solid black contours represent the $\mathrm{H}_{2}$ emission at $2.12 \mu \mathrm{m}$; dashed contours are the $1.4 \mathrm{~mm}$ continuum emission. Red and blue contours are the SMA integrated emission of the $\mathrm{SiO}(5-4)$ line $\left(v_{\mathrm{bl}}=[-30,-20] \mathrm{km} \mathrm{s}^{-1}\right.$ and $\left.v_{\mathrm{rd}}=[+10,+39] \mathrm{km} \mathrm{s}^{-1}\right)$. The crosses mark the Herschel pointings; the solid and dotted circles are the Herschel beams (Sect. 2). The square marks the peak of the EHV $\mathrm{CO}(2-1)$ red-shifted emission (R1). The arrow marks the OF1 outflow.

Herschel and the limited high angular resolution complementary data resulted in a lack of specific studies dedicated to outflows from massive YSOs.

The prominent far-IR source IRAS 17233-3606 (hereafter IRAS 17233) is one of the best laboratories for studying massive star formation because of its close distance (1 kpc, Leurini et al. 2011), high luminosity, and relatively simple geometry. In previous interferometric studies, we resolved three $\mathrm{CO}$ outflows with high collimation factors and extremely high velocity (EHV) emission (Leurini et al. 2009, Paper I). Their kinematic ages $\left(10^{2}-10^{3} \mathrm{yr}\right)$ point to deeply embedded YSOs that still have not reached the main sequence. One of the outflows, OF1 (Fig. 1), was the subject of a dedicated analysis in $\mathrm{SiO}$ lines (Leurini et al. 2013, Paper II). It is associated with $\mathrm{EHV} \mathrm{CO}(2-1), \mathrm{H}_{2}, \mathrm{SO}$, and $\mathrm{SiO}$ emission. $\mathrm{SiO}(5-4)$ and (8-7) APEX spectra suggest an increase of excitation with velocity and point to hot and/or dense gas close to the primary jet. Through a combined shock-LVG analysis of $\mathrm{SiO}$, we derived a mass of $>0.3 M_{\odot}$ for OF1, which implies a luminosity $L \geq 10^{3} L_{\odot}$ for its driving source.

In this Letter, we present observations of water towards IRAS 17233 with the HIFI instrument (de Graauw et al. 2010) onboard Herschel (Pilbratt et al. 2010).

\section{Observations}

Six water lines and one $\mathrm{H}_{2}^{18} \mathrm{O}$ transition were observed towards the positions $\alpha_{\mathrm{J} 2000}=17^{\mathrm{h}} 26^{\mathrm{m}} 42.50, \delta_{\mathrm{J} 2000}=-36^{\circ} 09^{\prime} 18^{\prime \prime} \cdot 00$ (OBSIDs 1342242862, 1342242863, and 1342242875), and $\alpha_{\mathrm{J} 2000}=17^{\mathrm{h}} 26^{\mathrm{m}} 42^{\mathrm{s}} .54, \delta_{\mathrm{J} 2000}=-36^{\circ} 09^{\prime} 20^{\prime} 00$ (OBSIDs 1342266457 and 1342266536) with a relative offset of $\left(0.5,-2{ }^{\prime}(0)\right.$. Conversion to $T_{\mathrm{mb}}$ was made using the beam efficiencies given in Table B.1 and a forward efficiency of 0.96 . Data were taken simultaneously in $\mathrm{H}$ and $\mathrm{V}$ polarisations using the acousto-optical Wide-Band Spectrometer. OBSIDs 1342242862,1342242863 , and 1342242875 were acquired in spectral scan mode with a redundancy of 4 to allow for sideband separation (Comito \& Schilke 2002). The data were calibrated with the standard calibration pipeline within HIPE 11.0 (Ott 2010). Sideband separation was performed using the GILDAS ${ }^{1}$

\footnotetext{
1 http://wWW.iram.fr/IRAMFR/GILDAS
}

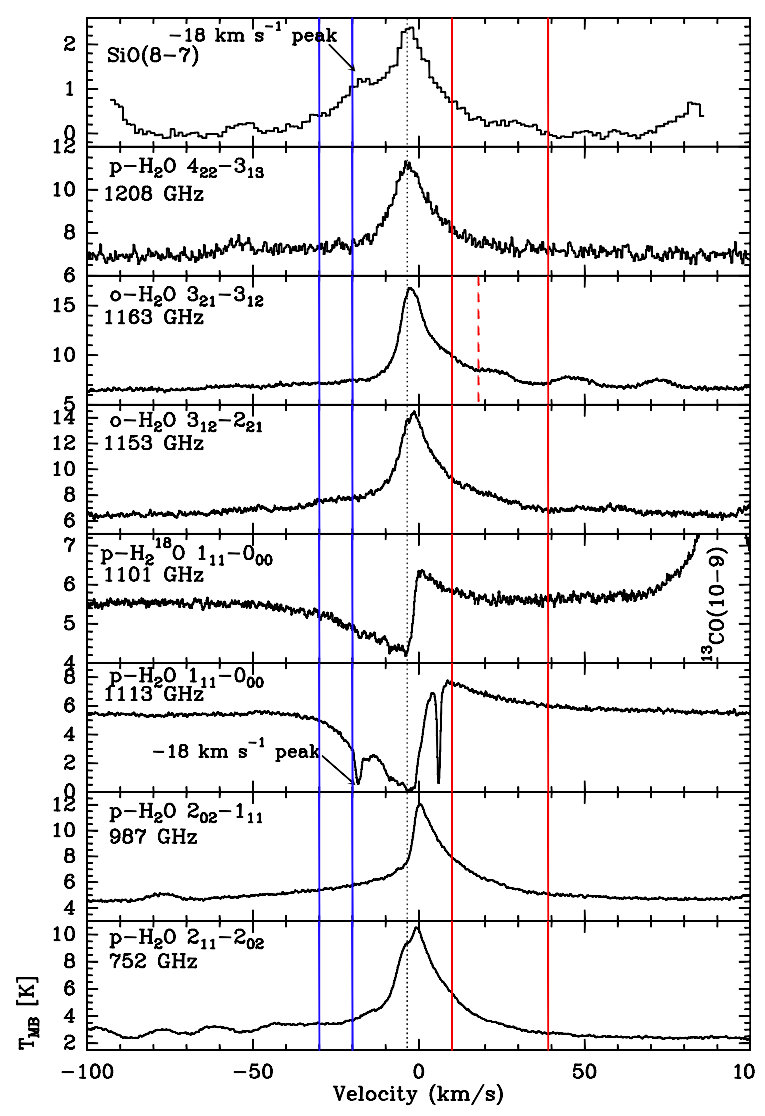

Fig. 2. Spectra of the water lines and of the $\mathrm{SiO}(8-7)$ transition towards IRAS 17233-3606. In all Herschel spectra, the continuum level is divided by a factor of two to correct for the fact that HIFI operates in double-sideband. The red and blue lines mark the velocity range used for the modelling of the water emission (solid red: $[+10,+39] \mathrm{km} \mathrm{s}^{-1}$; dashed red: $[+18] \mathrm{km} \mathrm{s}^{-1}$; blue: $[-30,-20] \mathrm{km} \mathrm{s}^{-1}$, Sect. 4). The $\mathrm{SiO}(8-7)$ spectrum has a beam size of $18^{\prime \prime}$, similar to the $19^{\prime \prime}$ beam size of the $1113 \mathrm{GHz}$ line, and it is observed at $\left(4 .{ }^{\prime \prime} 7,0{ }^{\prime} 0\right)$ from the Herschel $\alpha_{\mathrm{J} 2000}=17^{\mathrm{h}} 26^{\mathrm{m}} 42^{\mathrm{s}} .54, \delta_{\mathrm{J} 2000}=-36^{\circ} 09^{\prime} 20^{\prime} .00$ pointing. The dotted line marks the ambient velocity.

CLASS package. OBSIDs 1342266457 and 1342266536 were taken in single-pointing mode and level 2 data were exported into CLASS90 where they were analysed in detail. After inspection, data from the two polarisations were averaged together.

\section{Observational results}

Figure 2 shows the $\mathrm{H}_{2} \mathrm{O}$ spectra towards IRAS 17233. In all transitions, we detected water at high-velocities with respect to the ambient velocity $\left(v_{\mathrm{LSR}}=-3.4 \mathrm{~km} \mathrm{~s}^{-1}\right.$, Bronfman et al. 1996): indeed, IRAS 17233 presents one of the broadest profiles in the $1_{11}-0_{00}$ transition in high-mass YSOs (van der Tak et al. 2013) known to date. The ground-state line shows narrow absorptions at -18 and $+6 \mathrm{~km} \mathrm{~s}^{-1}$. They might be due to different clouds along the line of sight. However, the $\mathrm{SiO}(8-7)$ line, observed with a similar angular resolution (Paper II), has a well-defined emission peak at $-18 \mathrm{~km} \mathrm{~s}^{-1}$ (see Fig. 2) although broader than the $\mathrm{H}_{2} \mathrm{O}$ absorption. At -18 and $+6 \mathrm{~km} \mathrm{~s}^{-1}$ Zapata et al. (2008) detected $\mathrm{H}_{2} \mathrm{O}$ maser spots coming from the region shown in Fig. 1. These absorptions might be due to cold water associated with the outflows. The $\mathrm{H}_{2} \mathrm{O}$ and $\mathrm{H}_{2}^{18} \mathrm{O}$ ground-state lines have deep blue-shifted absorptions against the continuum and the outflow at velocities up to $-50 \mathrm{~km} \mathrm{~s}^{-1}$, while the main isotopologue line shows red-shifted emission up to $50 \mathrm{~km} \mathrm{~s}^{-1}$ 
and its $\mathrm{H}_{2}^{18} \mathrm{O}$ equivalent up to $+17 \mathrm{~km} \mathrm{~s}^{-1}$. High-velocity redshifted emission is detected up to $+50 / 60 \mathrm{~km} \mathrm{~s}^{-1}$ in all other lines, except in the highest energy line $\left(p-\mathrm{H}_{2} \mathrm{O} 4_{22}-3_{13}\right)$ where emission is detected only up to $+18 \mathrm{~km} \mathrm{~s}^{-1}$. The red-shifted wing of the $1163 \mathrm{GHz}$ line and the blue-shifted wing of the $752 \mathrm{GHz}$ transition are contaminated by hot-core-like features. Emission up to $-70 \mathrm{~km} \mathrm{~s}^{-1}$ is detected in the other transitions.

Comparison of the $\mathrm{H}_{2} \mathrm{O}$ and $\mathrm{H}_{2}^{18} \mathrm{O} 1_{11}-0_{00}$ profiles in the redwings shows that the main isotopologue line is deeply affected by absorption also at high velocities since red-shifted emission is detected from $1.3 \mathrm{~km} \mathrm{~s}^{-1}$ in $\mathrm{H}_{2}^{18} \mathrm{O}$ and only from $9 \mathrm{~km} \mathrm{~s}^{-1}$ in $\mathrm{H}_{2} \mathrm{O}$ (Fig. 2). The line ratio between the two $1_{11}-0_{00}$ isotopologue lines ranges between 0.95 and 0.3 in the blue wing $\left([-30,-20] \mathrm{km} \mathrm{s}^{-1}\right)$, establishing very high opacities for the main isotopologue transition even at high velocities and suggesting that it may be contaminated by a component in emission. Indeed, assuming negligible excitation with respect to the continuum, the opacity of the $\mathrm{H}_{2}^{18} \mathrm{O}$ line is between 0.02 and 0.3 in the velocity interval $[-50,-4] \mathrm{km} \mathrm{s}^{-1}$ (see Eq. (1) of Herpin et al. 2012). This corresponds to a column density of $\mathrm{H}_{2}^{18} \mathrm{O}$ of $8.4 \times 10^{11} \mathrm{~cm}^{-2}$ at the peak of the absorption, down to $5.5 \times$ $10^{10} \mathrm{~cm}^{-2}$ in the high-velocity wing $\left(-50 \mathrm{~km} \mathrm{~s}^{-1}\right)$. The total $\mathrm{p}-\mathrm{H}_{2}^{18} \mathrm{O}$ column density over the velocity range $[-50,-4] \mathrm{km} \mathrm{s}^{-1}$ is $1.2 \times 10^{13} \mathrm{~cm}^{-2}$. Assuming that the $1113 \mathrm{GHz}$ thermal continuum has the same distribution as at $1.4 \mathrm{~mm}$ (deconvolved size at FWHM of 5 !. $3 \times 2$ '.7, Paper I and Fig. 1), we corrected the continuum emission for beam dilution in the Herschel beam (Table B.1) and estimate a p- $\mathrm{H}_{2}^{18} \mathrm{O}$ column density of $2.4 \times$ $10^{14} \mathrm{~cm}^{-2}$, which corresponds to a total column density of $\mathrm{H}_{2} \mathrm{O}$ of $5.3 \times 10^{17} \mathrm{~cm}^{-2}$ for a standard isotopic ratio ${ }^{16} \mathrm{O} /{ }^{18} \mathrm{O}=560$ (Wilson \& Rood 1994) and a ortho-to-para ratio of 3. This is most likely a lower limit to the $\mathrm{H}_{2} \mathrm{O}$ column density since the $1113 \mathrm{GHz}$ thermal continuum is probably more compact than that at $1.4 \mathrm{~mm}$.

Given the complexity of the $1113 \mathrm{GHz}$ line at low-velocities, we focussed our analysis on the outflow component detected at high-velocities. The similarity of the $\mathrm{SiO}$ and $\mathrm{H}_{2} \mathrm{O}$ profiles suggests a common origin of the high-velocity emission in the two molecules. Therefore, we limited our analysis to the velocity ranges $[+10,+39] \mathrm{km} \mathrm{s}^{-1}$ and $[-30,-20] \mathrm{km} \mathrm{s}^{-1}$ used in Paper II. For the $1163 \mathrm{GHz}$ line, we used the velocity range $[+10,+18] \mathrm{km} \mathrm{s}^{-1}$. We did not include the $752 \mathrm{GHz}$ blue wing in the analysis because of severe contamination from other features.

\section{Shock-model of the water emission}

In Paper II, we demonstrated that the $\mathrm{SiO}$ emission in $\mathrm{OF} 1$ can be reproduced by a C-type shock model. We interpreted the $\mathrm{SiO}(8-7)$ and (5-4) emission at high velocities as due mostly $(\sim 60 \%)$ to the OF1 outflow and modelled their maximum brightness temperature and wing-integrated line ratio. Our best fit was found for a pre-shock density $n_{\mathrm{H}}=10^{6} \mathrm{~cm}^{-3}$, shock velocity $v_{\mathrm{s}}=32 \mathrm{~km} \mathrm{~s}^{-1}$, magnetic field strength $B=100 \mu \mathrm{G}$, and an age between 500 and $1000 \mathrm{yr}$, in agreement with observations (Paper I). The emitting area of the $\mathrm{SiO}(5-4)$ transition is similar to that of $\mathrm{H}_{2}, 6 \operatorname{arcsec}{ }^{2}$, with an upper limit of $22 \operatorname{arcsec}^{2}$. Our goal is to determine if the SiO-fitting shock can also reproduce the observed $\mathrm{H}_{2} \mathrm{O}$ emission. Since the $\mathrm{SiO}$ modelling was performed towards a position $\sim 9^{\prime \prime}$ off from the Herschel pointing, our first step was to verify that the model of Paper II is also valid on this position. We then post-processed the shock model with an LVG module to calculate the radiative transfer of water lines (Gusdorf et al. 2011). We thus compared modelled

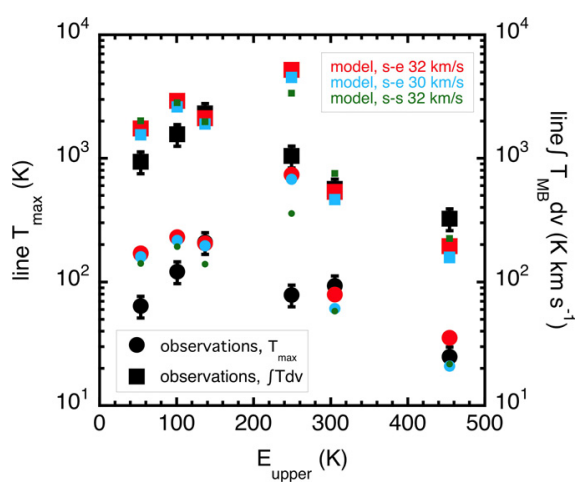

Fig. 3. Observed and modelled maximum brightness temperatures (circles), and integrated intensities (squares) for the red lobe of OF1. Data (in black) are corrected for an area of $6 \mathrm{arcsec}^{2}$, and for $60 \%$ of the emission due to OF1. Error bars are $\pm 20 \%$ of the observed values. Three models are shown: the model of Paper II with level populations in statistical equilibrium ("s-e" in red) with $v_{\mathrm{s}}=32 \mathrm{~km} \mathrm{~s}^{-1}$, one with a slower shock velocity $\left(v_{\mathrm{s}}=30 \mathrm{~km} \mathrm{~s}^{-1}\right.$, blue), and a model in stationary-state ("s-s" in green).

maximum brightness temperatures and integrated intensities to their observed values for two lines of $\mathrm{o}-\mathrm{H}_{2} \mathrm{O}$ and four lines of $\mathrm{p}-\mathrm{H}_{2} \mathrm{O}$, under the exact same assumptions as adopted for $\mathrm{SiO}$ : emitting area of $6 \operatorname{arcsec}^{2}$, with $60 \%$ of the emission due to the OF1 outflow. The results are in Figs. 3 and B.1, Tables B.2 and B.3. To provide an estimate on modelling uncertainties, we added the results of the radiative transfer computed in stationary state instead of statistical equilibrium (see Gusdorf et al. 2011, for details), and for a slightly slower shock model to account for the positional discrepancy between $\mathrm{SiO}$ and $\mathrm{H}_{2} \mathrm{O}$ observations. The $\mathrm{o}-\mathrm{H}_{2} \mathrm{O}$ line at $1153 \mathrm{GHz}$ is dramatically over-predicted by all models. However, this transition is masing in our LVG calculations (and in RADEX, van der Tak et al. 2007) and therefore predictions are not reliable. Three high-lying transitions are nicely reproduced in terms of maximum brightness temperature and integrated intensity in both the red- and blue-shifted component, although with a smaller area for the blue shifted case, $3 \operatorname{arcsec}^{2}$. Estimates of the $\mathrm{SiO}$ lines with this area are still compatible with the observations, and there is no other constraint on the area of the blue lobe since $\mathrm{H}_{2}$ is not detected. The lowenergy lines (p- $\mathrm{H}_{2} \mathrm{O}$ at 1113 and $988 \mathrm{GHz}$, Table B.1) are overpredicted by the model. Three explanations might be invoked to explain this discrepancy. First, these lines could be partly selfabsorbed even at the high-velocities used in our analysis. This could be true for the $1113 \mathrm{GHz}$ red-wing, as suggested by the sharp absorption at $+6 \mathrm{~km} \mathrm{~s}^{-1}$, directly at the edge of the velocity range used for the shock analysis, and by the comparison with $\mathrm{H}_{2}^{18} \mathrm{O} 1_{11}-0_{00}$ detected in emission at lower velocities than $\mathrm{H}_{2} \mathrm{O}$. However, there is no evidence for self-absorption in the $988 \mathrm{GHz}$ line. The optical thickness of these lines might also explain the discrepancy between models and data (non-local radiative transfer might affect their emissivity more than in the other lines). But the most convincing argument is that $\mathrm{H}_{2} \mathrm{O}$ could be dissociated in the quiescent parts of the shock, affecting the transitions that are most likely to emit in these regions. In this case, one should detect emission from the most abundant photodissociation products, namely $\mathrm{OH}$ and O (van Dishoeck 1988). Future observations with SOFIA might help to support this scenario. Refined shock-codes including effects of radiation fields are also needed to address this question.

If we accept that the $\mathrm{SiO}$ model also fits the $\mathrm{H}_{2} \mathrm{O}$ emission, we can infer the column density and the mass of $\mathrm{H}_{2} \mathrm{O}$ in OF1 because the column density is self-consistently computed in our 
shock model, and we have constraints on the area of the emission region. Whether we adopt an age of 500 or $1000 \mathrm{yr}$ (Paper II), the maximum $\mathrm{H}_{2} \mathrm{O}$ fractional abundance with respect to $n_{\mathrm{H}}$ in the shocked layer is $x_{\mathrm{H}_{2} \mathrm{O}} \simeq 1.4 \times 10^{-4}$. The corresponding water abundance in fractional column density units is $2.5 \times 10^{-5}$ for a dynamical age of $500 \mathrm{yr}$, and $1.2 \times 10^{-5}$ for an age of $1000 \mathrm{yr}$ (see Appendix A). The corresponding column density over the shock layer is $N_{\mathrm{H}_{2} \mathrm{O}}=1.2 \times 10^{18} \mathrm{~cm}^{-2}$, almost a factor of two higher than the lower limit $\left(5.3 \times 10^{17} \mathrm{~cm}^{-2}\right)$ found in Sect. 3 based on crude assumptions. For an area of $6 \operatorname{arcsec}^{2}$ for the red-lobe and of $3 \operatorname{arcsec}^{2}$ for the blue one, at $1 \mathrm{kpc}$ distance this column density corresponds to a shocked water mass of $3.8 \times 10^{-5} M_{\odot}$, or $12.5 M_{\oplus}$.

In our model, the maximum of the local $\mathrm{H}_{2} \mathrm{O}$ density is attained $45 \mathrm{yr}$ after the temperature peak. The highest value is a result of sputtering of the ices in the grain mantles, and of hightemperature chemistry. Because the sputtering is simultaneous to the temperature rise, $45 \mathrm{yr}$ is the time scale for the hightemperature chemistry under these shock conditions. Given the small $\mathrm{O}_{2}$ abundance measured in dense cold molecular clouds, water is mainly formed via the sputtering of grain mantles, for which standard models predict a total release of material towards the gas phase above a shock velocity threshold of $20-25 \mathrm{~km} \mathrm{~s}^{-1}$ (e.g., Draine et al. 1983; Flower \& Pineau des Forets 1994). Since both shock velocities used in our analysis are well above the threshold shock speed for water, the derived $\mathrm{H}_{2} \mathrm{O}$ abundance does not change significantly at $v_{\mathrm{s}}=30 \mathrm{~km} \mathrm{~s}^{-1}$.

\section{Discussion and conclusions}

The $\mathrm{SiO}(8-7)$ and $\mathrm{H}_{2} \mathrm{O}$ profiles (in particular that of the $1113 \mathrm{GHz}$ line) suggest a common origin of the $\mathrm{H}_{2} \mathrm{O}$ and $\mathrm{SiO}$ emission in IRAS 17233. This result is based on emission at high velocities and is different from the findings that $\mathrm{SiO}$ and $\mathrm{H}_{2} \mathrm{O}$ do not trace the same gas in molecular outflows from low-mass YSOs at low-velocities and/or in low-energy lines (Santangelo et al. 2012; Nisini et al. 2013). However, an excellent match between $\mathrm{SiO}$ and $\mathrm{H}_{2} \mathrm{O}$ profiles is found in other sources at high velocities (Lefloch et al. 2012).

With the limitations previously discussed, we find that the shock parameters of OF1 are comparable with those found for low-mass protostars with a higher pre-shock density. The derived water abundance is compatible with values of other molecular outflows (e.g., Emprechtinger et al. 2010; Herczeg et al. 2012). While often measurements of $\mathrm{H}_{2} \mathrm{O}$ abundances have large uncertainties because the $\mathrm{H}_{2}$ column density is inferred from observations of $\mathrm{CO}$ or from models (for a compilation of sources, abundances and methods, see van Dishoeck et al. 2013), the value inferred in our analysis is consistently derived, as the $\mathrm{H}_{2} \mathrm{O}$ and $\mathrm{H}_{2}$ column densities are outcomes of the same model. Moreover, the estimated $\mathrm{H}_{2} \mathrm{O}$ column density matches the data. Although photo-dissociation probably affects the lowenergy $\mathrm{H}_{2} \mathrm{O}$ lines, simple $\mathrm{C}$-shocks models can be used to model higher-energy transitions. The inclusion of photo-dissociation in our models is work in progress in a larger framework of studying the effect of an intense UV field on shocks.

Estimates of $\mathrm{H}_{2} \mathrm{O}$ mass are not easily found in the literature. Busquet et al. (2014) modelled water emission in L1157-B1 through J- and C-type shocks. Their $\mathrm{H}_{2} \mathrm{O}$ column densities derived over the whole line profiles translate in to masses in the range $0.009-0.125 M_{\oplus}$ for a hot component of $2^{\prime \prime}-5^{\prime \prime}$ size and $<(0.7-1.5) 10^{-3} M_{\oplus}$ for a warm component with a size of $\leq 10^{\prime \prime}$. Our estimate of $12.5 M_{\oplus}$ for the $\mathrm{H}_{2} \mathrm{O}$ mass of $\mathrm{OF} 1$ therefore seems to be compatible with previous results.

In summary, we presented the first estimate of the abundance of water in an outflow driven by a massive YSOs based on a self-consistent shock model of water and $\mathrm{SiO}$ transitions. We inferred a water abundance in fractional column density units between $1.2 \times 10^{-5}$ and $2.5 \times 10^{-5}$, which is an average value of the water abundance over the shock layer. Additionally, our model indicates that the maximum fractional abundance of water locally reached in the layer is $10^{-4}$. Finally, we inferred the water mass of the OF1 outflow to be $12.5 M_{\oplus}$.

Acknowledgements. Herschel is an ESA space observatory with science instruments provided by European-led Principal Investigator consortia and with important participation from NASA. HIFI has been designed and built by a consortium of institutes and university departments from across Europe, Canada and the United States under the leadership of SRON Netherlands Institute for Space Research, Groningen, The Netherlands and with major contributions from Germany, France and the US. Consortium members are: Canada: CSA, U.Waterloo; France: CESR, LAB, LERMA, IRAM; Germany: KOSMA, MPIfR, MPS; Ireland: NUI Maynooth; Italy: ASI, IFSI-INAF, Osservatorio Astrofisico di Arcetri- INAF; Netherlands: SRON, TUD; Poland: CAMK, CBK; Spain: Observatorio Astronómico Nacional (IGN), Centro de Astrobiología (CSIC-INTA). Sweden: Chalmers University of Technology - MC2, RSS \& GARD; Onsala Space Observatory; Swedish National Space Board, Stockholm University - Stockholm Observatory; Switzerland: ETH Zurich, FHNW; USA: Caltech, JPL, NHSC. A.G. acknowledges support by the grant ANR-09-BLAN0231-01 from the French Agence Nationale de la Recherche as part of the SCHISM project. T.Cs. is funded by the ERC Advanced Investigator Grant GLOSTAR (247078). A.G. acknowledges useful discussions with C. Vastel and A. Coutens.

\section{References}

Beuther, H., Schilke, P., Sridharan, T. K., et al. 2002, A\&A, 383, 892 Bronfman, L., Nyman, L.-A., \& May, J. 1996, A\&AS, 115, 81 Busquet, G., Lefloch, B., Benedettini, M., et al. 2014, A\&A, 561, A120 Comito, C., \& Schilke, P. 2002, A\&A, 395, 357

de Graauw, T., Helmich, F. P., Phillips, T. G., et al. 2010, A\&A, 518, L6 Draine, B. T., Roberge, W. G., \& Dalgarno, A. 1983, ApJ, 264, 485 Emprechtinger, M., Lis, D. C., Bell, T., et al. 2010, A\&A, 521, L28 Flower, D. R., \& Pineau des Forets, G. 1994, MNRAS, 268, 724 Flower, D. R., \& Pineau Des Forêts, G. 2010, MNRAS, 406, 1745 Gusdorf, A., Giannini, T., Flower, D. R., et al. 2011, A\&A, 532, A53 Herczeg, G. J., Karska, A., Bruderer, S., et al. 2012, A\&A, 540, A84 Herpin, F., Chavarría, L., van der Tak, F., et al. 2012, A\&A, 542, A76 Kahn, F. D. 1974, A\&A, 37, 149

Kaufman, M. J., \& Neufeld, D. A. 1996, ApJ, 456, 611

Kristensen, L. E., van Dishoeck, E. F., Bergin, E. A., et al. 2012, A\&A, 542, A8 Krumholz, M. R., \& Bonnell, I. A. 2009, Models for the formation of massive stars, ed. G. Chabrier (Cambridge University Press), 288

Lefloch, B., Cabrit, S., Busquet, G., et al. 2012, ApJ, 757, L25

Leurini, S., Codella, C., Zapata, L. A., et al. 2009, A\&A, 507, 1443 (Paper I) Leurini, S., Codella, C., Zapata, L., et al. 2011, A\&A, 530, A12

Leurini, S., Codella, C., Gusdorf, A., et al. 2013, A\&A, 554, A35 (Paper II)

Nisini, B., Santangelo, G., Antoniucci, S., et al. 2013, A\&A, 549, A16 Ott, S. 2010, in Astronomical Data Analysis Software and Systems XIX, eds. Y. Mizumoto, K.-I. Morita, \& M. Ohishi, ASP Conf. Ser., 434, 139

Pickett, H. M., Poynter, I. R. L., Cohen, E. A., et al. 1998, J. Quant. Spectr. Radiat. Transf., 60, 883

Pilbratt, G. L., Riedinger, J. R., Passvogel, T., et al. 2010, A\&A, 518, L1 Roelfsema, P. R., Helmich, F. P., Teyssier, D., et al. 2012, A\&A, 537, A17 Santangelo, G., Nisini, B., Giannini, T., et al. 2012, A\&A, 538, A45 Tafalla, M., Liseau, R., Nisini, B., et al. 2013, A\&A, 551, A116 van der Tak, F. F. S., Black, J. H., Schöier, F. L., Jansen, D. J., \& van Dishoeck, E. F. 2007, A\&A, 468, 627

van der Tak, F. F. S., Chavarría, L., Herpin, F., et al. 2013, A\&A, 554, A83 van Dishoeck, E. F. 1988, in Rate Coefficients in Astrochemistry, eds. T. J. Millar, \& D. A. Williams, Astrophys. Space Sci. Lib., 146, 49 van Dishoeck, E. F., Kristensen, L. E., Benz, A. O., et al. 2011, PASP, 123, 138 van Dishoeck, E. F., Herbst, E., \& Neufeld, D. A. 2013, Chem. Rev., 113, 9043 Wilson, T. L., \& Rood, R. 1994, ARA\&A, 32, 191

Zapata, L. A., Leurini, S., Menten, K. M., et al. 2008, AJ, 136, 1455

Pages 5 to 7 are available in the electronic edition of the journal at http://www . aanda. org 


\section{Appendix A: Water abundance problem: the point of view of observers and modellers}

The goal of this appendix is to clarify the possible confusion of the meaning of "water abundance" between the observing and modelling communities. The rigorous comparison of observations to models requires the knowledge of constraints such as the length/age of the shock, as this section discusses now. We base this discussion on the model used to fit both the $\mathrm{SiO}$ and $\mathrm{H}_{2} \mathrm{O}$ emission in the OF1 shock region of IRAS 17233-3606 with the following input parameters: pre-shock density $n_{\mathrm{H}}=10^{6} \mathrm{~cm}^{-3}$, shock velocity $v_{\mathrm{s}}=32 \mathrm{~km} \mathrm{~s}^{-1}$, and magnetic field strength (perpendicular to the shock direction) $B=1 \mathrm{mG}$. Whether the radiative transfer of water is calculated along the shock equations in the model (so-called "s-s" in Fig. 3, "DRF" in Tables B.2-B.5) or a posteriori from the outputs of the shock model ("s-e" in Fig. 3, "AGU" in Tables B.2-B.5) does not change the thermal profile of the shock layer, nor the associated water abundances (e.g. Gusdorf et al. 2011). Everything stated in this appendix is therefore applicable to both "s-s" and "s-e" models.

In one-dimensional, stationary shock models (e.g., this work, Gusdorf et al. 2011; Draine et al. 1983; Kaufman \& Neufeld 1996; Flower \& Pineau Des Forêts 2010) the physical and chemical conditions are self-consistently calculated at each point of a shocked layer. The end product is a collection of physical (temperature, velocity, density) and chemical (abundances) quantities obtained at each point of the shocked layer. The position of each point is marked by a distance parameter with respect to a origin typically located in the pre-shock region. The position of the last point in the post-shock region then corresponds to the shock width. Typically, these shock models are used in a face-on configuration, so that the width one refers to is along the lineof-sight direction. Alternatively, the position of a point in the shock layer can be expressed through a time parameter: the time parameter for the last point in the post-shock region then corresponds to the flight time that a particle needs to flow through the total width of the shock. The correspondence between the time and distance parameters related to a neutral particle $\left(t_{\mathrm{n}}\right.$ and $\left.z\right)$ is hence given by $t_{\mathrm{n}}=\int\left(1 / v_{\mathrm{n}}\right) \mathrm{d} z$, where $v_{\mathrm{n}}$ is the particle velocity. While the shock width cannot be constrained by observations, an upper limit to the flow time is given by the dynamical age, which is inferred from mapped observations of spectrally resolved lines.

Figure A. 1 shows for this model the variation of the temperature of the neutral particles $(\mathrm{K})$, as well as those of the water and total local densities $\left(n\left(\mathrm{H}_{2} \mathrm{O}\right)\right.$ and $n_{\text {tot }}$ in $\left.\mathrm{cm}^{-3}\right)$ and their ratio $x\left(\mathrm{H}_{2} \mathrm{O}\right)=n\left(\mathrm{H}_{2} \mathrm{O}\right) / n_{\text {tot }}$ in the shock layer versus the distance parameter. To illustrate the relation between time and distance parameters through the shock layer, we have marked three points on each curve: $3.1 \times 10^{15}, 5.15 \times 10^{15}, 10^{16} \mathrm{~cm}$, which correspond to 500, 1000, and $2150 \mathrm{yr}$, in our model. In our case, the highest value for the time parameter is constrained by the dynamical shock age of OF1, 500-1000yr. Water abundance is often defined by modellers as the maximum fractional local abundance of water through the shock layer, that is, between the pre-shock region before the temperature rise and the maximum shock age $\left(x\left(\mathrm{H}_{2} \mathrm{O}\right)_{\max }=1.4 \times 10^{-4}\right.$ for our model, top panel of Fig. A.1). On the other hand, local quantities cannot be accessed through observations. Integrated quantities (against the width of the shock layer along the line of sight) such as column densities are measured by observers. Generally, "observational water abundances" are hence given in fractional column density units, that is, the ratio of the water column density divided by the total column density. This ratio is different the maximum fractional
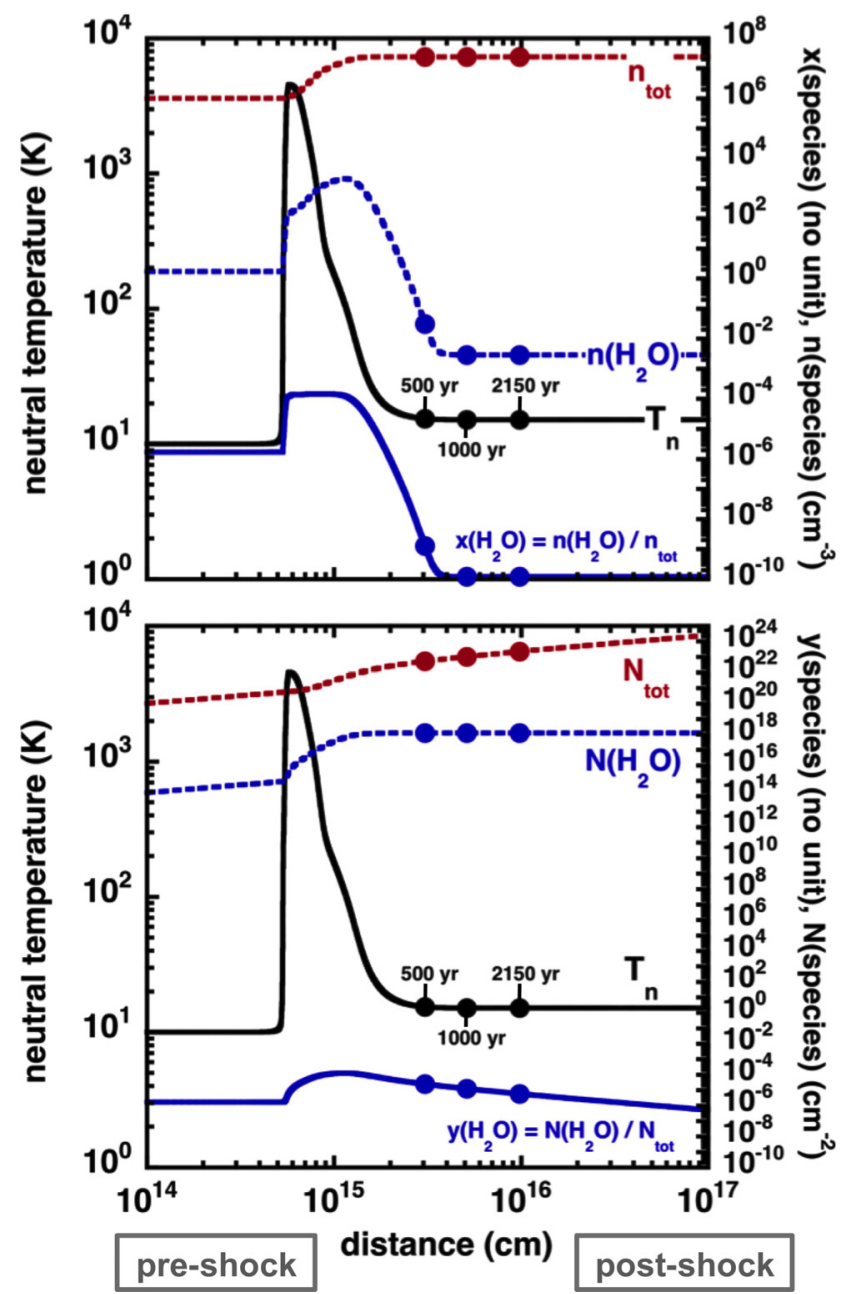

Fig. A.1. Upper panel: neutral temperature (black curve), total density (red dashed curve), water density (blue dashed curve), and fractional density (blue continuous curve). The so-called fractional density is the water density over the total density, locally defined at each point of the shock. Lower panel: neutral temperature (black curve), total column density (red dashed curve), water column density (blue dashed curve), and fractional column density (blue continuous curve). The so-called fractional column density is the water column density over the total column density. The column density (in $\mathrm{cm}^{-2}$ ) is the integral of the local density (in $\mathrm{cm}^{-3}$ ) along the shock width (in $\mathrm{cm}$ ). In both panels, the three points labelled on each curve correspond to the distance parameter of $3.1 \times 10^{15}, 5.15 \times 10^{15}, 10^{16} \mathrm{~cm}$, or to time parameters values of 500 , 1000 , and $2150 \mathrm{yr}$.

abundance of water that is generally provided and used by modellers. The difference between the two values is illustrated by comparing the upper panel of Fig. A.1 with its lower panel, which shows the evolution of the water and total column densities, $N_{\mathrm{H}_{2} \mathrm{O}}$ and $N_{\text {tot }}$, and of their ratio $y\left(\mathrm{H}_{2} \mathrm{O}\right)=N\left(\mathrm{H}_{2} \mathrm{O}\right) / N_{\text {tot }}$. In the modellers' view, referring to the distance parameter as " $z$ ", these column densities are defined by

$$
\begin{aligned}
& N\left(\mathrm{H}_{2} \mathrm{O}\right)\left[\mathrm{cm}^{-2}\right]=\int_{0}^{\mathrm{z}_{\max }} n\left(\mathrm{H}_{2} \mathrm{O}\right)\left[\mathrm{cm}^{-3}\right] \mathrm{d} z, \\
& N_{\text {tot }}\left[\mathrm{cm}^{-2}\right]=\int_{0}^{\mathrm{z}_{\max }} n_{\text {tot }}\left[\mathrm{cm}^{-3}\right] \mathrm{d} z,
\end{aligned}
$$

where $z_{\max }$ is the total shock width, that is, the distance corresponding to the maximum value of the time parameter. In our case, the value of the fractional column density of water can be 
read in the bottom panel of Fig. A.1: $y\left(\mathrm{H}_{2} \mathrm{O}\right)=2.5 \times 10^{-5}$ (if the adopted dynamical age is $500 \mathrm{yr}$ ),$=1.2 \times 10^{-5}$ (if the adopted dynamical age is $1000 \mathrm{yr}$ ). We note that this value is about an order of magnitude lower than the maximum fractional abundance of water reached in the same shock layer.

We note that the decrease in the $y\left(\mathrm{H}_{2} \mathrm{O}\right)$ curve is artificial and only due to the 1D nature of the model. Indeed, in the postshock region, the total density of the gas is conserved (because it cannot escape sideways, for instance like in the case of a bowshock), while the gas-phase water density decreases until all water molecules re-condensate on the interstellar grains because of the temperature decrease. The total column density hence increases (lower panel of Fig. A.1), while the water column density is constant, resulting in a decrease of the water column density ratio with the distance or time parameter. It is therefore essential to have a measurement of the dynamical time scale to stop the calculation at a realistic time to obtain a fractional column density of water as realistic as possible.

\section{Appendix B: Additional tables and figures}

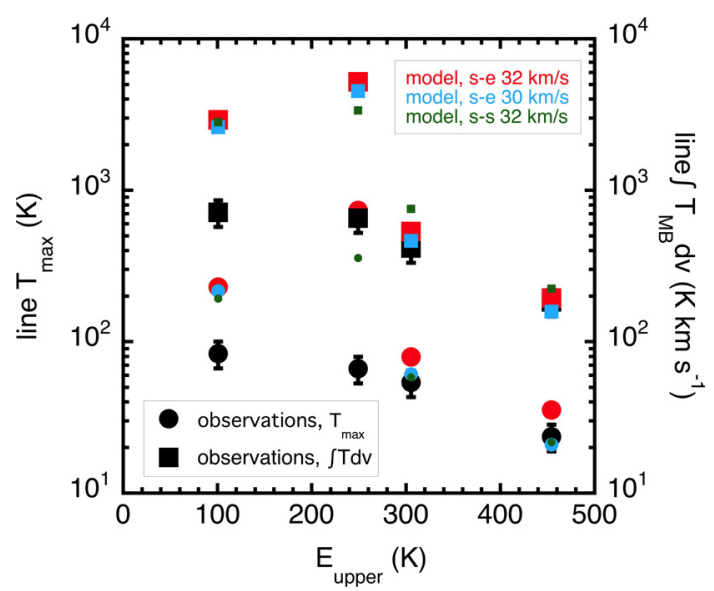

Fig. B.1. Observed and modelled maximum brightness temperatures (circles), and integrated intensities (squares) for the blue-shifted emission. Data points (in black) are corrected for an emission region of $3 \operatorname{arcsec}^{2}$ and for $60 \%$ of the emission due to OF1. Errorbars are $\pm 20 \%$ of the observed value. Three models are shown: the model of Paper II with level populations in statistical equilibrium ("s-e" in red) with $v_{\mathrm{s}}=32 \mathrm{~km} \mathrm{~s}^{-1}$, one with a slower shock velocity $\left(v_{\mathrm{s}}=30 \mathrm{~km} \mathrm{~s}^{-1}\right.$, blue), and a model in stationary-state ("s-s" in green).

Table B.1. Summary of the observations.

\begin{tabular}{|c|c|c|c|c|c|c|c|c|c|}
\hline Line & $\begin{array}{l}v^{1} \\
(\mathrm{GHz})\end{array}$ & $\begin{array}{l}E_{\text {up }}^{1} \\
(\mathrm{~K})\end{array}$ & $\begin{array}{l}\text { Beam }^{2} \\
\left({ }^{\prime \prime}\right)\end{array}$ & $\eta_{\mathrm{mb}}^{2}$ & $\begin{array}{l}T_{\text {sys }} \\
(\mathrm{K})\end{array}$ & $\begin{array}{c}\delta v \\
\left(\mathrm{~km} \mathrm{~s}^{-1}\right)\end{array}$ & $\begin{array}{l}\mathrm{rms} \\
(\mathrm{K})\end{array}$ & OBSIDs & $\operatorname{mode}^{3}$ \\
\hline$p-\mathrm{H}_{2} \mathrm{O} 4_{22}-3_{13}$ & 1207.639 & 454.5 & 17.6 & 0.64 & 1063 & 0.12 & 0.21 & 1342242862 & DBS \\
\hline$o-\mathrm{H}_{2} \mathrm{O} 3_{21}-3_{12}$ & 1162.912 & 305.4 & 18.2 & 0.64 & 850 & 0.13 & 0.18 & 1342242863 & DBS \\
\hline$o-\mathrm{H}_{2} \mathrm{O} 3_{12}-2_{21}$ & 1153.127 & 249.5 & 18.3 & 0.64 & 836 & 0.13 & 0.18 & 1342242863 & DBS \\
\hline$p-\mathrm{H}_{2} \mathrm{O} 1_{11}-0_{00}$ & 1113.343 & 53.5 & 19.0 & 0.74 & 389 & 0.10 & 0.13 & 1342266536 & DBS \\
\hline$p-\mathrm{H}_{2}^{18} \mathrm{O} 1_{11}-0_{00}$ & 1101.698 & 52.9 & 19.0 & 0.74 & 389 & 0.10 & 0.13 & 1342266536 & DBS \\
\hline$p-\mathrm{H}_{2} \mathrm{O} 2_{02}-1_{11}$ & 987.927 & 100.9 & 21.5 & 0.74 & 333 & 0.15 & 0.15 & 1342242875 & DBS \\
\hline$p-\mathrm{H}_{2} \mathrm{O} 2_{11}-2_{02}$ & 752.033 & 137.0 & 28.2 & 0.74 & 187 & 0.20 & 0.20 & 1342266457 & DBS \\
\hline
\end{tabular}

Notes. ${ }^{(1)}$ Pickett et al. (1998). ${ }^{(2)}$ Half-power beam width and main beam efficiency from Roelfsema et al. (2012). ${ }^{(3)}$ DBS stands for dual beam switch mode.

Table B.2. Observed and modelled maximum line temperatures $\left(T^{\max }, \mathrm{K}\right)$ for the red lobe.

\begin{tabular}{|c|c|c|c|c|c|c|c|c|}
\hline $\begin{array}{r}v \\
(\mathrm{GHz})\end{array}$ & $\begin{array}{l}E_{\mathrm{up}} \\
(\mathrm{K})\end{array}$ & $\begin{array}{c}\text { Beam } \\
\left({ }^{\prime \prime}\right)\end{array}$ & $\begin{array}{l}F F^{-1(1)} \\
\text { (no unit) }\end{array}$ & $\begin{array}{c}T_{\text {obs }}^{\max } \\
(\mathrm{K})\end{array}$ & $\begin{array}{c}T_{\text {obs,corr }}^{\max }(2) \\
(\mathrm{K})\end{array}$ & $\begin{array}{c}T_{\mathrm{AGU} 32}^{\max }(3) \\
(\mathrm{K})\end{array}$ & $\begin{array}{c}T_{\mathrm{AGU} 30}^{\max }(4) \\
(\mathrm{K})\end{array}$ & $\begin{array}{c}T_{\mathrm{DRF} 32}^{\max }(5) \\
(\mathrm{K})\end{array}$ \\
\hline 1113 & 53.4 & 19.1 & 48.5 & 2.2 & 64.0 & 169.8 & 159.1 & 141.5 \\
\hline 988 & 100.8 & 21.5 & 61.4 & 3.3 & 121.6 & 229.3 & 215.9 & 192.6 \\
\hline 752 & 136.9 & 28.2 & 105.1 & 3.3 & 208.1 & 204.3 & 195.1 & 139.8 \\
\hline 1153 & 249.3 & 18.4 & 45.3 & 2.9 & 78.8 & 735.9 & 676.1 & 356.9 \\
\hline 1163 & 305.3 & 18.2 & 44.5 & 3.5 & 93.5 & 79.4 & 61.0 & 58.1 \\
\hline 1208 & 454.3 & 17.6 & 41.4 & 1.0 & 24.8 & 35.4 & 20.9 & 21.7 \\
\hline
\end{tabular}

Notes. ${ }^{(1)}$ Inverse of the beam filling factor at each frequency considering an emitting area of $6 \operatorname{arcsec}^{2}$ (2) $^{(2)}$ Observed maximum temperature corrected for filling factor and $60 \%$ contribution of OF1. ${ }^{(3)}$ Modelled maximum temperature following Gusdorf et al. (2011) with $v_{\mathrm{s}}=32 \mathrm{~km} \mathrm{~s}{ }^{-1}$. (4) Modelled maximum temperature following Gusdorf et al. (2011) with $v_{\mathrm{s}}=30 \mathrm{~km} \mathrm{~s}^{-1}$. ${ }^{(5)}$ Modelled maximum temperature following Flower \& Pineau Des Forêts (2010) with $v_{\mathrm{s}}=32 \mathrm{~km} \mathrm{~s}^{-1}$. 
Table B.3. Observed and modelled integrated intensities $\left(\int T \mathrm{~d} v, \mathrm{~K} \mathrm{~km} \mathrm{~s}^{-1}\right)$ for the red lobe.

\begin{tabular}{rcccccccc}
\hline \hline $\begin{array}{r}v \\
(\mathrm{GHz})\end{array}$ & $\begin{array}{c}E_{\mathrm{up}} \\
(\mathrm{K})\end{array}$ & $\begin{array}{c}\text { Beam } \\
\left({ }^{\prime \prime}\right)\end{array}$ & $\begin{array}{c}F F^{-1(1)} \\
(\text { no unit) }\end{array}$ & $\begin{array}{c}{\left[\int T \mathrm{~d} v\right]_{\mathrm{obs}}} \\
\mathrm{K} \mathrm{km} \mathrm{s}^{-1}\end{array}$ & $\begin{array}{c}{\left[\int T \mathrm{~d} v\right]_{\mathrm{corr}^{(2)}}} \\
\mathrm{K} \mathrm{km} \mathrm{s}^{-1}\end{array}$ & $\begin{array}{c}{\left[\int T \mathrm{~d} v\right]_{\mathrm{AGU} 32}{ }^{(3)}} \\
\mathrm{K} \mathrm{km} \mathrm{s}^{-1}\end{array}$ & $\begin{array}{c}{\left[\int T \mathrm{~d} v\right]_{\mathrm{AGU} 30}{ }^{(4)}} \\
\mathrm{K} \mathrm{km} \mathrm{s}^{-1}\end{array}$ & $\begin{array}{c}{\left[\int T \mathrm{~d} v\right]_{\mathrm{DRF32} 2}{ }^{(5)}} \\
\mathrm{K} \mathrm{km} \mathrm{s}^{-1}\end{array}$ \\
\hline 1113 & 53.4 & 19.1 & 48.5 & 32.0 & 931.42 & 1743.0 & 1547.0 & 2006.7 \\
988 & 100.8 & 21.5 & 61.4 & 42.4 & 1562.1 & 2914.0 & 2606.0 & 2810.5 \\
752 & 136.9 & 28.2 & 105.1 & 36.6 & 2307.5 & 2112.0 & 1894.0 & 1976.3 \\
1153 & 249.3 & 18.4 & 45.3 & 38.4 & 1043.7 & 5188.0 & 4512.0 & 3366.6 \\
1163 & 305.3 & 18.2 & 44.5 & 21.1 & 563.4 & 534.6 & 462.5 & 752.1 \\
1208 & 454.3 & 17.6 & 41.4 & 13.0 & 322.9 & 194.2 & 158.0 & 223.8 \\
\hline
\end{tabular}

Notes. ${ }^{(1)}$ Inverse of the beam filling factor at each frequency considering an emitting area of $6 \operatorname{arcsec}^{2} .^{(2)}$ Observed integrated intensity corrected for filling factor and $60 \%$ contribution of OF1. ${ }^{(3)}$ Modelled integrated intensity following Gusdorf et al. (2011) with $v_{\mathrm{s}}=32 \mathrm{~km} \mathrm{~s}{ }^{-1}$. ${ }^{(4)}$ Modelled integrated intensity following Gusdorf et al. (2011) with $v_{\mathrm{s}}=30 \mathrm{~km} \mathrm{~s}^{-1}$. ${ }^{(5)}$ Modelled integrated intensity following Flower \& Pineau Des Forêts (2010) with $v_{\mathrm{s}}=32 \mathrm{~km} \mathrm{~s}^{-1}$.

Table B.4. Observed and modelled maximum line temperatures $\left(T^{\max }, \mathrm{K}\right)$ for the blue lobe.

\begin{tabular}{rcccccccc}
\hline \hline $\begin{array}{r}v \\
(\mathrm{GHz})\end{array}$ & $\begin{array}{c}E_{\text {up }} \\
(\mathrm{K})\end{array}$ & $\begin{array}{c}\text { Beam } \\
\left({ }^{\prime \prime}\right)\end{array}$ & $\begin{array}{c}F F^{-1(1)} \\
(\text { no unit })\end{array}$ & $\begin{array}{c}T_{\text {obs }}^{\max } \\
(\mathrm{K})\end{array}$ & $\begin{array}{c}T_{\text {obs,corr }}^{\max }(\mathrm{K}) \\
(121.8\end{array}$ & $\begin{array}{c}T_{\text {AGU32 }}^{\max }(3) \\
(\mathrm{K})\end{array}$ & $\begin{array}{c}T_{\text {AGU30 }}^{\max }(4) \\
(\mathrm{K})\end{array}$ & $\begin{array}{c}T_{\mathrm{DRF32}}^{\max }(5) \\
(\mathrm{K})\end{array}$ \\
\hline 988 & 100.8 & 21.5 & 121.8 & 1.1 & 83.3 & 229.3 & 215.9 & 192.6 \\
1153 & 249.3 & 18.4 & 89.5 & 1.2 & 66.3 & 735.9 & 676.1 & 356.9 \\
1163 & 305.3 & 18.2 & 88.0 & 1.0 & 53.9 & 79.4 & 61.0 & 58.1 \\
1208 & 454.3 & 17.6 & 81.8 & 0.5 & 23.6 & 35.4 & 20.9 & 21.7 \\
\hline
\end{tabular}

Notes. ${ }^{(1)}$ Inverse of the beam filling factor at each frequency considering an emitting area of $3 \operatorname{arcsec}^{2} .^{(2)}$ Observed maximum temperature corrected for filling factor and $60 \%$ contribution of OF1. ${ }^{(3)}$ Modelled maximum temperature following Gusdorf et al. (2011) with $v_{\mathrm{s}}=32 \mathrm{~km} \mathrm{~s}{ }^{-1}$. (4) Modelled maximum temperature following Gusdorf et al. (2011) with $v_{\mathrm{s}}=30 \mathrm{~km} \mathrm{~s}^{-1}$. ${ }^{(5)}$ Modelled maximum temperature following Flower \& Pineau Des Forêts (2010) with $v_{\mathrm{s}}=32 \mathrm{~km} \mathrm{~s}^{-1}$.

Table B.5. Observed and modelled integrated intensities $\left(\int T \mathrm{~d} v, \mathrm{~K} \mathrm{~km} \mathrm{~s}^{-1}\right)$ for the blue lobe.

\begin{tabular}{rcccccccc}
\hline \hline $\begin{array}{r}v \\
(\mathrm{GHz})\end{array}$ & $\begin{array}{c}E_{\mathrm{up}} \\
(\mathrm{K})\end{array}$ & $\begin{array}{c}\text { Beam } \\
\left({ }^{\prime \prime}\right)\end{array}$ & $\begin{array}{c}F F^{-1(1)} \\
(\text { no unit })\end{array}$ & $\begin{array}{c}{\left[\int T \mathrm{~d} v\right]_{\mathrm{obs}}} \\
\mathrm{K} \mathrm{km} \mathrm{s}^{-1}\end{array}$ & $\begin{array}{c}{\left[\int T \mathrm{~d} v\right]_{\mathrm{corr}}{ }^{(2)}} \\
\mathrm{K} \mathrm{km} \mathrm{s}^{-1}\end{array}$ & $\begin{array}{c}{\left[\int T \mathrm{~d} v\right]_{\mathrm{AGU} 32}{ }^{(3)}} \\
\mathrm{K} \mathrm{km} \mathrm{s}^{-1}\end{array}$ & $\begin{array}{c}{\left[\int T \mathrm{~d} v\right]_{\mathrm{AGU} 30}(4)} \\
\mathrm{K} \mathrm{km} \mathrm{s}^{-1}\end{array}$ & $\begin{array}{c}{\left[\int T \mathrm{~d} v\right]_{\mathrm{DRF} 32}{ }^{(5)}} \\
\mathrm{K} \mathrm{km} \mathrm{s}^{-1}\end{array}$ \\
\hline 988 & 100.8 & 21.5 & 121.8 & 9.8 & 714.9 & 2914.0 & 2606.0 & 2810.5 \\
1153 & 249.3 & 18.4 & 89.5 & 12.2 & 653.3 & 5188.0 & 4512.0 & 3366.6 \\
1163 & 305.3 & 18.2 & 88.0 & 7.9 & 415.1 & 534.6 & 462.5 & 752.1 \\
1208 & 454.3 & 17.6 & 81.8 & 3.7 & 183.1 & 194.2 & 158.0 & 223.8 \\
\hline
\end{tabular}

Notes. ${ }^{(1)}$ Inverse of the beam filling factor at each frequency considering an emitting area of $3 \operatorname{arcsec}^{2} .^{(2)}$ Observed integrated intensity corrected for filling factor and $60 \%$ contribution of OF1. (3) Modelled integrated intensity following Gusdorf et al. (2011) with $v_{\mathrm{s}}=32 \mathrm{~km} \mathrm{~s}^{-1}$. ${ }^{(4)}$ Modelled integrated intensity following with $v_{\mathrm{s}}=30 \mathrm{~km} \mathrm{~s}^{-1}{ }^{(5)}$ Modelled integrated intensity following Flower \& Pineau Des Forêts (2010) with $v_{\mathrm{s}}=$ $32 \mathrm{~km} \mathrm{~s}^{-1}$. 